\title{
Ventricular fibrillation in a 20-year old patient with non- ischemic cardiomyopathy aborted by wearable cardioverter defibrillator
}

\author{
Nicolas W Shammas ${ }^{1}$, Yuhning Linda $\mathrm{Hu}^{2}$, Peter Sharis ${ }^{2}$, Renae VanZuiden ${ }^{2}$ and Ellen Holm ${ }^{2}$ \\ ${ }^{1}$ Research Director, Midwest Cardiovascular Research Foundation, USA \\ ${ }^{2}$ Cardiovascular Medicine, PC and the Midwest Cardiovascular Research Foundation, USA
}

\begin{abstract}
The use of Wearable Cardioverter Defibrillator (WCD) has been controversial in patients diagnosed with non-ischemic cardiomyopathy prior to permanent internal defibrillator implantation. We present a case of a 20 -year old patient presented with severe non-ischemic cardiomyopathy. He was started on conventional medical treatment and was discharged home with a LifeVest ${ }^{\circledR}$ (ZOLL, Pittsburgh, PA, USA). The next am he sustained a ventricular fibrillation arrest and was defibrillated successfully with the LifeVest ${ }^{\circledR}$. This case illustrates the importance of protecting patients with a LifeVest ${ }^{\circledR}$ when they have a severe reduction in ejection fraction using a WCD irrespective of age or the lack of coronary artery disease.
\end{abstract}

\section{Introduction}

LifeVest $^{\oplus}$ (ZOLL, Pittsburgh, PA, USA) is a wearable cardioverter defibrillator (WCD) designed to protect patients at risk of sudden cardiac death (SCD). It is a temporary device aimed at protecting patients while recovering from an original insult that led to the severe reduction in their ejection fraction (EF) [1]. Patients with non-ischemic cardiomyopathy or post myocardial infarction severe left ventricular dysfunction are likely to be suitable candidates for this device [2-5]. However, there has been significant debate among cardiologists to the cost-effectiveness of this device in patients with non-ischemic cardiomyopathy considering that SCD is relatively rare in these patients in the first few months after their initial insult.

\section{Case presentation}

We present the case of a 20 -year-old male who presented with dyspnea on exertion for one month. He also noted chest tightness that has been progressive along with his dyspnea. He had no immediate preceding illness but did have the flu a year prior. He has been healthy otherwise. Upon presentation to the emergency room, he was in atrial fibrillation with a rapid ventricular response. He received metoprolol intravenously and started on a Cardizem drip. His BP was $89 / 56$ $\mathrm{mmHg}$ and he was $96 \%$ on 5 liters of oxygen by nasal cannula. He had negative troponin I but his BNP was elevated to $6089 \mathrm{ng} / \mathrm{ml}$. TSH was normal. ESR was $1 \mathrm{~mm} /$ hour. His electrocardiogram showed rapid atrial fibrillation with nonspecific lateral $\mathrm{T}$ waves abnormalities and septal Q waves. His CXR showed moderate pleural effusion, mild pulmonary edema and mild cardiac enlargement. He does not smoke and drinks alcohol occasionally. His exam was remarkable for his irregular heart beat and diminished breath sounds over the lung bases. He had no JVD and no lower extremity edema. Computed tomography angiography (CTA) of the chest showed no pulmonary embolus. His echocardiogram showed an EF of less than $20 \%$ and moderate mitral regurgitation. Angiography was performed and showed his coronaries to be normal with no coronary anomalies. Transesophageal echocardiogram was performed and showed no left atrial thrombus. Cardioversion was performed successfully with restoration of normal sinus rhythm.

Patient was initiated on carvedilol, spironolactone and furosemide. His blood pressure was too low to initiate an angiotensin blocker. Because of his atrial fibrillation and his CHADs score of 1 , he was placed on aspirin. The working diagnosis was viral cardiomyopathy or tachycardia-induced cardiomyopathy. He was fitted with a LifeVest and discharged home. The next day, the patient experienced a ventricular fibrillation episode, successfully detected by the LifeVest and he was defibrillated back into sinus rhythm (Figure 1A and B). This recurred once. Patient was then transferred to a University Hospital for further care upon his request.

\section{Discussion}

LifeVest ${ }^{\oplus}$ is indicated for patients in the recovery phase of their severe cardiomyopathy. In a study of 8453 patients with post myocardial infarction severe reduction in left ventricular function, the WCD successfully treated SCA in $1.4 \%$ of patients, and the risk was highest in the first month of WCD use [2]. Also, in the WEARIT-II Registry that enrolled 2000 patients with ischemic, nonischemic cardiomyopathy, or congenital/inherited heart disease, the rate of sustained ventricular arrhythmias by 90 days was $3 \%$ among ischemic cardiomyopathy

Correspondence to: Nicolas W Shammas, Research Director, Midwest Cardiovascular Research Foundation, 1622 E Lombard Street, Davenport, IA 52803, USA, E-mail: shammas@mchsi.com

Key words: Ventricular fibrillation, non-ischemic cardiomyopathy, Wearable Cardioverter defibrillator, cardiac arrest, young patient

Received: February 15, 2018; Accepted: March 05, 2018; Published: March 10 2018 

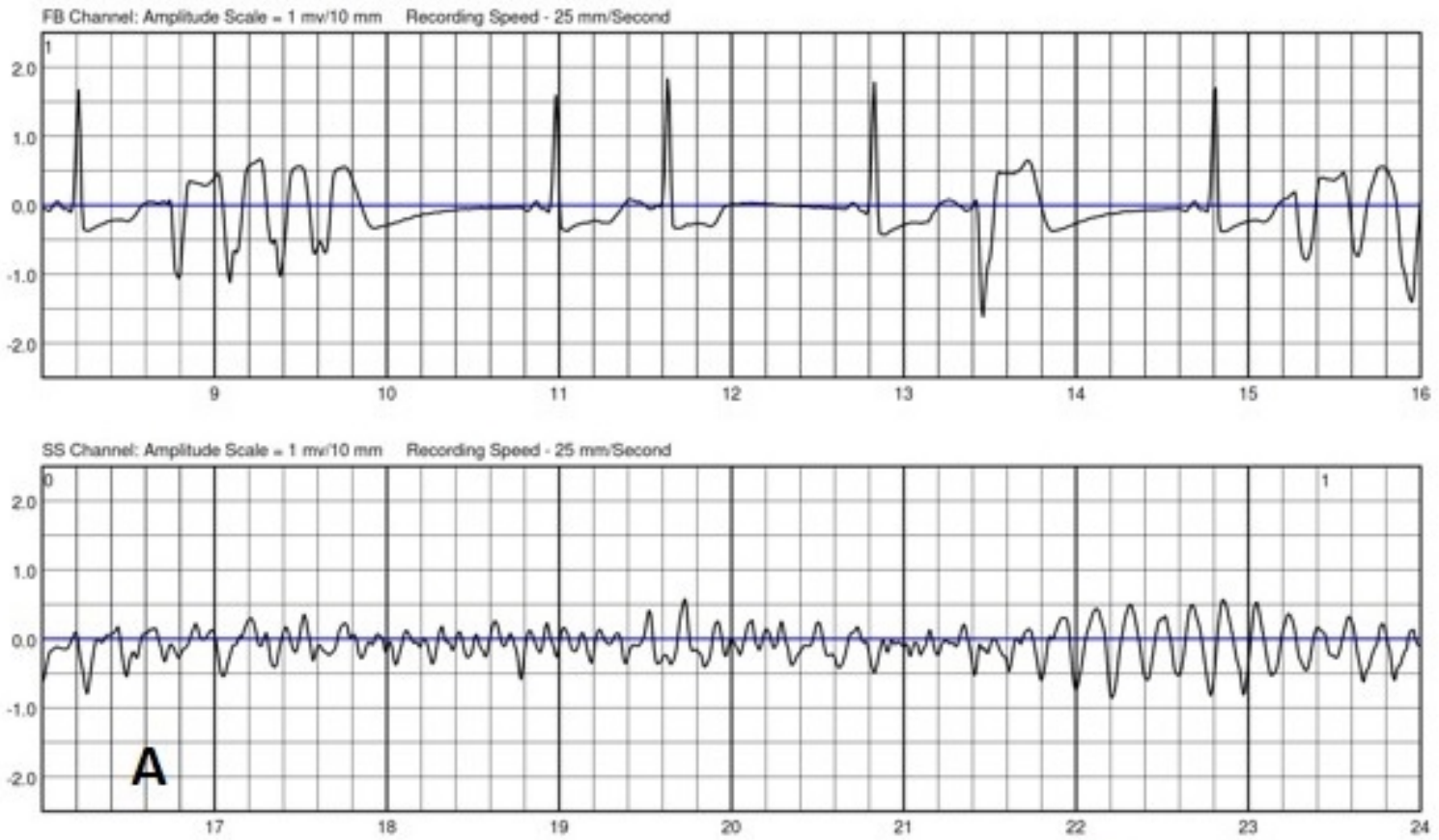

FB Channel: Amplitude Scale - 1 mw10 mm Peconding Speed - 25 mmsecond
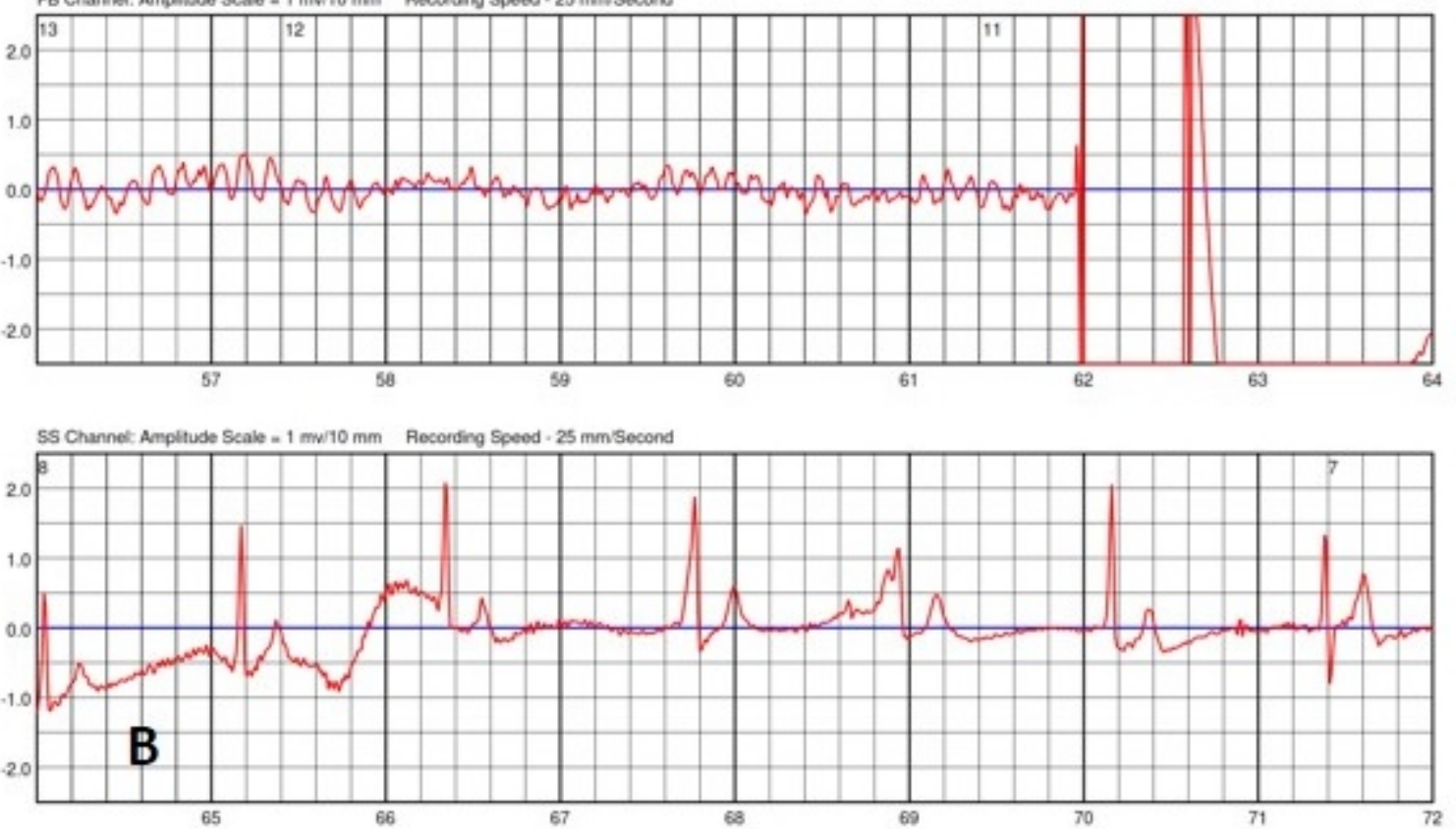

Figure 1. Episode of ventricular fibrillation (1A) that was successfully defibrillated (1B) by the LifeVest.

patients and $1 \%$ among non-ischemic patients [3]. At the end of WCD use, $42 \%$ were implanted with a permanent implantable cardioverter defibrillator because of continued reduced below $35 \% \mathrm{EF}$. In addition, recent studies have shown that the WCD can be utilized as a bridge to transplantation in patients with severe LV dysfunction [4]. Although no randomized trials have been performed with the WCD, registries indicate there is a potential benefit of this device in saving lives. However, there is considerable debate about placing a LifeVest in a non-ischemic cardiomyopathy patient awaiting permanent ICD and while in the optimization phase of his/her medical therapy. It is argued that that these patients have rare ventricular fibrillation episodes during this waiting phase and LifeVest cost-effectiveness cannot justify its routine use. In our 20-year-old patient the LifeVest did save his life. Although it may be a rare event, it is difficult to predict which nonischemic CM patient will experience ventricular fibrillation. Also, the device is temporary prior to transition to a permanent ICD if necessary. It requires minimal learning and it is easy to wear. We believe this device is essential in patients with newly diagnosed severe $(<35 \%$ ejection fraction) non-ischemic cardiomyopathy and can prove to be a lifesaving intervention. Future research continues to be needed to confirm its value in this patient population. 


\section{Conflict of interest}

Renae VanZuiden is an employee of Zoll Medical. None of the other authors have a relevant conflict of Interest to this case

\section{References}

1. Klein HU, Goldenberg I, Moss AJ (2013) Risk stratification for implantable cardioverter defibrillator therapy: the role of the wearable cardioverter-defibrillator. Eur Heart J 34: 2230-42 [Crossref]

2. Epstein AE, Abraham WT, Bianco NR, Kern KB, Mirro M, et al. (2013) Wearable cardioverter-defibrillator use in patients perceived to be at high risk early postmyocardial infarction. J Am Coll Cardiol 62: 2000-2007 [Crossref]
3. Kutyifa V, Moss AJ, Klein H, Biton Y, McNitt S, et al. (2015) Use of the wearable cardioverter defibrillator in high-risk cardiac patients: data from the Prospective Registry of Patients Using the Wearable Cardioverter Defibrillator (WEARIT-II Registry). Circulation 132: 1613-1619 [Crossref]

4. Opreanu M, Wan C, Singh V, Salehi N, Ahmad J, et al. (2015) Wearable cardioverterdefibrillator as a bridge to cardiac transplantation: A national database analysis. J Heart Lung Transplant. 34: 1305-1309. [Crossref]

5. Feldman AM, Klein H, Tchou P, Murali S, Hall WJ, et al. (2004) WEARIT investigators and coordinators; BIROAD investigators and coordinators. Use of a wearable defibrillator in terminating tachyarrhythmias in patients at high risk for sudden death: results of the WEARIT/BIROAD. Pacing Clin Electrophysiol 27: 4-9 [Crossref]

Copyright: (C2018 Shammas NW. This is an open-access article distributed under the terms of the Creative Commons Attribution License, which permits unrestricted use, distribution, and reproduction in any medium, provided the original author and source are credited. 